\title{
A Hybrid Method to Diagnose 3D Rotor Eccentricity Faults in Synchronous Generators Based on ALIF_PE and KFCM
}

\author{
Chao Zhang $\mathbb{D}^{1},{ }^{1}$ Chuang-Jin He, ${ }^{1}$ Shuai Li, ${ }^{1}$ Yu-Ling He ${ }^{D},{ }^{1}$ Xiao-Long Wang $\left(\mathbb{D},{ }^{1}\right.$ \\ Xiang-Yu Liu $\mathbb{D}{ }^{2}$ and Lun Cheng ${ }^{3}$ \\ ${ }^{1}$ Department of Mechanical Engineering and Hebei Key Laboratory of Electric Machinery Health Maintenance and \\ Failure Prevention, North China Electric Power University, Baoding 071003, China \\ ${ }^{2}$ State Grid Hebei Electric Power Research Institute, Shijiazhuang 050021, China \\ ${ }^{3}$ State Grid Hebei Electric Power Supply Co., Ltd., Shijiazhuang 050022, China \\ Correspondence should be addressed to Yu-Ling He; heyuling1@163.com
}

Received 5 February 2021; Revised 3 May 2021; Accepted 24 May 2021; Published 1 June 2021

Academic Editor: Francisco Beltran-Carbajal

Copyright ( 92021 Chao Zhang et al. This is an open access article distributed under the Creative Commons Attribution License, which permits unrestricted use, distribution, and reproduction in any medium, provided the original work is properly cited.

This paper proposed a new hybrid diagnosis method for the generator's 3D static eccentricity faults which include the axial eccentricity, the radial eccentricity, and the mixed eccentricity composed of the former two. Firstly, adaptive local iterative filtering (ALIF) method was used to decompose the vibration signals of the generator under eccentricity faults. Then, in order to figure out the intrinsic mode function (IMF) components with the upmost feature information, the correlation coefficient was calculated. Finally, the components' permutation entropy $(\mathrm{PE})$ is extracted to construct the eigenvector matrix which can be used to input the kernel fuzzy C-means (KFCM) algorithm to obtain the result of clustering. The result indicates that the classification coefficient based on ALIF and KFCM behaves closer to 1, while the average fuzzy entropy (FE) is closer to 0, showing that this method is able to detect different eccentricity faults more accurately.

\section{Introduction}

As the center of electric system, generator is a typical highspeed rotating machine which is highly potential to suffer different faults such as rotor eccentricity or other mechanical failures from time to time $[1,2]$. In order to realize the early detection and avoid losses, it is significant to explore highefficiency methods in particular for each fault.

The typical faults of generator include rotor short circuit, stator short circuit, and air-gap eccentricity. Among them, eccentricity fault will lead to the change of air-gap magnetic field which would worsen the performances of generator and even damage the machine as a result of vibration, rotor bending, winding wear, and friction between stator and rotor [3]. Therefore, eccentricity fault detection is one of the important contents of generator fault diagnosis.

To detect the eccentricity faults, there are two common views: motor current signature analysis and vibration signals
[4]. Some scholars pay attention to the motor current signature analysis; for example, Taner Goktas et al. [5] dealt with the discernment of broken magnet and static eccentricity faults through the stator phase current. They analyzed stator electromotive force and phase current waveforms in detail to identify the discerning components and characterize their dynamic behaviors. The method was verified through both simulations and experiments. Attoui and Omeiri [6] proposed a new fractional-order controller (FOC) with a simple and practical design method which can ensure the stability of the nonlinear system in both healthy and faulty conditions. And they used an online fault diagnostic technique based on the spectral analysis of stator currents by a fast Fourier transform (FFT) algorithm in order to detect the stator and rotor faults.

Besides, the fault diagnosis methods based on the vibration signal are widely used in rolling bearing. However, it is also effective and worth researching in generator because it 
can help us extract the fault feature. If we want to realize fault classification based on vibration signal, the study of signal processing technique is essential. In recent years, many scholars have been widely exploring the signal processing method. Encouragingly, a series of achievements have been obtained. For instance, in 1998, Huang et al. [7] proposed the empirical mode decomposition (EMD) method which can get the amplitude and frequency of signal by decomposing the nonstationary signal into several intrinsic mode function (IMF) components and combining with Hilbert transform.

However, about the EMD method, the problem of mode mixing is inevitable so that researchers spare no effort to overcome it. In 2009, Wu et al. [8,9] combined the EMD method with the features of white Gaussian noise whose frequency distribution is uniform to solve this problem efficiently and they proposed ensemble empirical mode decomposition (EEMD) whose essence is to decompose the nonlinear and nonstationary multimodal signal from high frequency to low frequency into IMF components. The method shows a good decomposition effect. Xue et al. [10] used EEMD to decompose the ground-penetrating radar (GPR) signal into a series of IMFs and calculated the permutation entropy (PE) of each IMF to distinguish noise IMFs and target IMFs. Finally, they reconstructed the signal with target IMFs to remove the noise effectively.

Based on the research of the former scholars, M. A. Torres et al. [11] proposed complete ensemble empirical mode decomposition with adaptive noise (CEEMDAN) method which can add white noise to the signal adaptively according to the features and obtain the IMF components by calculating the only residual signal, so as to solve the problem of mode mixing and improve the efficiency of decomposition. It is widely introduced in the field of fault diagnosis. For instance, in order to solve the problem that the fault feature extraction is difficult because the vibration signal of rolling bearing is polluted by strong noise, Ji and Wang [12] proposed a CEEMDAN aided fast spectral kurtosis graph algorithm for fault diagnosis. The method decomposed the signal into multimodal components using the CEEMDAN algorithm and reconstructed the effective component by kurtosis value. The processing result of the algorithm indicated that the fault detection of bearing inner ring can be realized effectively.

With the purpose of realizing a better decomposition effect, people have also tried alternative methods. Cicone et al. [13] proposed the method of adaptive local iterative filtering (ALIF) based on the method of iterative filtering (IF) in 2016. In the process of iterative decomposition, the filter function of ALIF can alter different expressions with the signal changes; thus, the multimode signal can be decomposed into multiorder single components adaptively. Given its advantages, Tang and Pang [14] used ALIF to decompose the rotor fault vibration signal of turbogenerator sets to obtain several IMF components and then obtained the instantaneous frequency and amplitude of each IMF component through Hilbert transform. Finally, the fault type of the shafting was identified according to the time-frequency features of rotor vibration.
In order to represent the complexity of signal, entropy theory, including fuzzy entropy (FE), sample entropy (SE), $\mathrm{PE}$, and approximate entropy (AE), is proposed and widely used in many fields of data analysis such as energy, material, and physiology $[15,16]$. For example, Cao and Lin [17] proposed using inherent FE and its multiscale version, which employs EMD and fuzzy membership function to address the dynamic complexity in electroencephalogram (EEG) data.

In recent years, scholars have been trying to apply the entropy theory in fault diagnosis. As is known, feature extraction is one of the most important issues in mechanical fault diagnosis, which directly relates to the accuracy and the reliability of early fault prediction. Zhao et al. [18] used the EEMD method to decompose the vibration signal of bearing in generator and the correlation coefficient analysis method to determine three improved IMFs, which are close to the original signal. Then, they used the multiscale fuzzy theory to calculate the entropy values of the selected three IMFs in order to form a feature vector with the complexity measure, which is regarded as the inputs of the support vector machine (SVM) model for training and constructing an SVM classifier to recognize fault pattern. Their method can extract much feature information and effectively eliminate the impact of mode mixing. In 2016, Rostaghi and Azami [19] proposed dispersion entropy (DE), which not only has fast calculation speed but also is less affected by the mutation signal and takes into account the difference between the signal amplitude. As its application in generator, Wang et al. [20] distinguished the rotor short circuit and stator short circuit based on variational mode decomposition (VMD) and the refined composite multiscale dispersion entropy (RCMDE).

To characterize the complexity of the signal and quantify the signal features, in this paper, the PE algorithm is introduced which is proposed by Bandt and Pompe [21] to detect randomness and dynamic mutation. It has simple calculation, strong antinoise ability, and high sensitivity to signal changes. Subsequently, Ding and Zhang [22] used the Haar wavelet and PE method to denoise and extract fault eigenvalues. Then, they classified different gear faults according to the principle of different $\mathrm{PE}$ distribution corresponding to different faults. Moreover, Ren et al. [23] screened several inherent frequency band functions obtained by decomposing the hydrogenerator signal to achieve the purpose of denoising.

For further research, it is necessary to classify the fault feature information to realize the detection of different types of faults. Clustering is to classify a group of data with unknown distribution, merge data with the same properties into the same class or cluster, and divide data with different properties into different classes or clusters $[24,25]$. The traditional fuzzy C-means (FCM) algorithm is relatively mature; on this basis, the kernel learning process was introduced in kernel fuzzy C-means (KFCM) algorithm which stuck out the feature differences by mapping the samples to high-dimensional space [26]. As for its application, Bi et al. [27] used VMD to decompose the signal of diesel engine and then selected the key 
component whose maximum singular value would be calculated and input to KFCM to realize the classification of the faults of diesel engine. In addition, Zhang et al. [28] proposed a novel classifier that combined rough sets and SVM in the fault diagnosis for hydroelectric generator unit (HGU). They extracted the fault patterns lying in the overlapped region instead of classifying the patterns directly. Then, they defined the upper and lower approximations of each class on the basis of the rough set technique. Next, they calculated the reliability that they belong to a certain class for the fault patterns lying in the overlapped region. The results show that the proposed classifier can more properly describe the complex map between the faults and their symptoms.

With the development of artificial intelligence (AI) technology, some scholars spare no efforts to introduce AI technology to the field of fault classification; for example, Gao et al. [29] realized the classification of faults in rotor-bearing systems through AI model, which needs sufficient fault samples to have a better effect. They combined the finite element method (FEM) with generative adversarial networks (GANs) for rotor-bearing systems to expand fault samples so as to improve the accuracy. In addition, Wang et al. [30] proposed a method using deep belief networks (DBN) to detect multiple faults in axial piston pumps. For each individual fault, they calculated all the data indicators of the signals to construct samples. Then, they input the samples into DBNs to classify the multiple faults. With restricted Boltzmann machine (RBM) stacked layer by layer, DBNs can automatically learn fault features. These methods embodied the trend of intellectualization and automation.

Honestly, we suppose that the fault diagnosis would be more and more intelligent in the future. At the same time, clustering algorithm in fault diagnosis will probably develop in full swing. On the basis of this perspective, we proposed a new method that combines the PE method with ALIF and KFCM to meet the complicated feature extraction of nonlinear dynamics in generators in this paper. In fact, eccentricity fault is often not one-dimensional so that the diagnosis method focused on 3D faults shows huge potential. Besides, some other methods about eccentricity faults such as [31] focused on the degree of eccentricity faults rather than the type. Therefore, the method in this paper contributes ideas to diagnosis of the fault types just like the axial eccentricity, the radial eccentricity, and the mixed eccentricity composed of the former two. As presented in the following, the samples representing the same fault type are divided into a vivid cluster and we can easily recognize different fault types of generator.

The remainder of this paper is constructed as follows. The theoretical model and process of the proposed method are presented in Section 2 and Section 3, respectively, while the simulation and the experimental study of the proposed method are illustrated in Section 4. The results analysis is in Section 5. Finally, the brief conclusions drawn from the study are presented in Section 6.

\section{Theoretical Model of the Proposed Method}

The proposed method includes three parts, namely, the noise filtering based on ALIF, the PE calculation of the decomposed signal, and the clustering based on KFCM.

2.1. ALIF Filter. The filter function of ALIF can alter different expressions with the signal changes; thus, the multimode signal can also be decomposed into multiorder components adaptively [18]. The process of the ALIF algorithm includes inner loop and outer loop.

The function of the inner loop is to select each IMF component iteratively. The moving operator $\Gamma(z(t))$ can be obtained by

$$
\Gamma(z(t))=\int_{-l(z)}^{l(z)} z(t+\tau) \omega(\tau) \mathrm{d} \tau,
$$

where $\omega(t)$ is the filtering function and $l(z)$ is the filtering interval which can be written as

$$
l(z)=2\left[\frac{N \lambda}{m}\right],
$$

where $\lambda \in(1.6,2), m$ is the number of extreme points, and $N$ is the signal length.

The solution process of $\omega(t)$ is as follows.

Suppose that $h(x)$ and $g(x)$ are smooth differentiable functions and satisfy the following conditions on the interval $[a, b]$ :

(1) $g(a)=g(b)=0, g(x)>0$, for any $x \in(a, b)$

(2) $h(a)<0<h(b)$

Then, the general form of the Fokker Planck equation is

$$
\frac{\partial p}{\partial t}=-\delta \frac{\partial(h(x) p)}{\partial x}+\mu \frac{\partial^{2}\left(g^{2}(x) p\right)}{\partial x^{2}},
$$

where the value range of $\delta$ and $\mu$ is $(0,1)$.

In (3), $\partial^{2}(g(x) p) / \partial x^{2}$ makes the solution $p(x)$ of the equation from the midpoint of the interval $(a, b)$ to the endpoints $a$ and $b$, and $-\partial(h(x) p) / \partial x$ makes $p(x)$ gather from the endpoints $a$ and $b$ to the center of the interval.

When the two mentioned are in balance, it has

$$
-\delta \frac{\partial(h(x) p)}{\partial x}+\mu \frac{\partial^{2}\left(g^{2}(x) p\right)}{\partial x^{2}}=0 .
$$

And the solution $p(x)$ of the equation now would be the filtering function $\omega(t)$, which satisfies

(1) $p(x) \geq 0$ for any $x \in(a, b)$

(2) $p(x)=0$ for any $x \notin(a, b)$

The wave operator $\kappa(z(t))$ can be obtained by subtracting the original signal $z(t)$ by the moving operator $\Gamma(z$ $(t))$ as

$$
\kappa(z(t))=z(t)-\Gamma(z(t)) .
$$

However, the iterative process will not be going on forever. Equation (6) is usually the condition to stop the 
process. When $E_{i}$ is less than the specified threshold, the inner loop iterative filtering will stop.

$$
E_{i}=\frac{\left\|\kappa_{i, n}-\kappa_{i, n-1}\right\|_{2}}{\left\|\kappa_{i, n-2}\right\|_{2}} .
$$

The effect of outer loop is to stop the process of IMF extraction by inner loop.

When all IMF components are removed from the original signal $z(t)$ and the residual $r(t)$ shows obvious trend features, the outer loop will stop. The detailed process is illustrated in Figure 1.

2.2. Permutation Entropy. Permutation entropy is an appropriate complexity measure for chaotic time series, in particular in the presence of dynamical and observational noise [21].

Given its obvious superiority in reflecting small abrupt change behavior of system vibration response, $\mathrm{PE}$ is suitable for signal of mechanical equipment. The fundamental principle of the algorithm is as follows.

Construct a set of time series $\left\{x_{i} \mid i=1,2, \ldots, N\right\}$; then, reconstruct the phase space. It has

$$
X_{i}=\left[x_{i}, x_{i}+\tau, \ldots, x_{i}+(m-1) \tau\right] .
$$

where $m$ is the embedding dimension and $\tau$ is the delay time.

Any $X_{i}$ has $m$ ! permutations. For any permutation $w, T$ $(w)$ represents the number of times it appears; then, the probability of its occurrence is

$$
P(w)=\frac{T(w)}{N-(m-1) \tau} .
$$

Thus, PE can be defined as

$$
H_{\mathrm{PE}}=-\sum P(w) \ln P(w) .
$$

After normalization, it has

$$
\mathrm{PE}=\frac{H_{\mathrm{PE}}}{\ln (m !)}
$$

The size of PE reflects the complexity and the randomness of the time-series signal. The larger PE means a more complicated time-series signal. The dimension $m$ and the delay time $\tau$ will impact the calculation result during extracting the features by PE. If $m$ is too small, the reconstructed vector contains too few states so that the algorithm will lose sense. However, if $m$ is too large, the reconstruction of the phase space would homogenize the time series which would lead to long-time calculation and fewer details. The influence of the delay time $\tau$ is relatively smaller [21].

2.3. KFCM Cluster. Kernel fuzzy $c$-means (KFCM) clustering is a method to map the samples which are classified into high-dimensional space through nonlinear mapping of kernel space and then cluster after highlighting the differences of samples [31]. The nonlinear map $\Phi$ is defined as

$$
\Phi_{n} x_{k} \longrightarrow \Phi\left(x_{k}\right) \in F
$$

where $x_{k}$ is the original feature space sample, $x_{k} \in X$.

The clustering objective function of the KCFM algorithm can be expressed as

$$
J_{m}=\sum_{i=1}^{c} \sum_{k=1}^{n} \mu_{i k}^{m}\left\|\Phi\left(x_{k}\right)-\Phi\left(v_{i}\right)\right\|^{2},
$$

where $v_{i}$ is the clustering center, $c$ is the number of clustering, $n$ is the number of samples, $\mu_{i k}$ is the degree of membership, and $m$ is the fuzzy weighted index.

At the same time, there is

$$
\begin{aligned}
& \mu_{i k} \in[0,1], \quad 0<\sum_{k=1}^{n} \mu_{i k}<n,(i=1, \ldots, c), \\
& \sum_{i=1}^{c} \mu_{i k}=1, \quad(k=1,2 n \operatorname{Ln} n),
\end{aligned}
$$

where $K(x, y)=\Phi^{T}(x) \Phi(y)$ and can be further written as

$$
K(x, y)=\exp \left[-\frac{\|x-y\|^{2}}{\left(2 \sigma^{2}\right)}\right],
$$

where $\sigma$ is the Gaussian kernel parameter. Then, the Euclidean distance of kernel space is

$$
\left\|\Phi\left(x_{k}\right)-\Phi\left(v_{i}\right)\right\|^{2}=K\left(x_{k}, x_{k}\right)+K\left(v_{i}, v_{i}\right)-2 K\left(x_{k}, v_{i}\right) \text {. }
$$

According to equations (14) and (15) as well as the constraint condition, the degree of the membership and the clustering center can be calculated by

$$
\begin{aligned}
u_{i k} & =\frac{\left[1-K\left(x_{k}, v_{i}\right)\right]^{-(1 /(m-1))}}{\sum_{j=1}^{c}\left[1-K\left(x_{k}, v_{j}\right)\right]^{-(1 /(m-1))}}, \\
v_{i} & =\frac{\sum_{k=1}^{n} \mu_{i k}^{m} K\left(x_{k}, v_{i}\right) x_{k}}{\sum_{k=1}^{n} \mu_{i k}^{m} K\left(x_{k}, v_{i}\right)} .
\end{aligned}
$$

To analyze the clustering effect, the classification coefficient $S$ and the average FE $E$ are presented, which are defined, respectively, as

$$
\begin{aligned}
& S=\frac{1}{n} \sum_{i=1}^{c} \sum_{j=1}^{n} u_{i j}^{2}, \\
& E=-\frac{1}{n} \sum_{i}^{c} \sum_{j}^{n} u_{i j} \ln u_{i j},
\end{aligned}
$$

where $c$ is the number of categories, $n$ is the number of samples, and $u_{i j}$ is the degree of membership.

The classification coefficient $S$ is used to express the fuzziness degree of the clustering result. If $S=1$, the clustering result belongs to the hard partition; else if $S<1$, it belongs to the fuzzy partition. Therefore, the closer $S$ is to 1 , the better the effect will be. The average FE indicates the uncertainty of the classification; for the hard partition, $E=0$, while for the fuzzy partition, $E>0$. Therefore, the effect will be better as $E$ moves closer to 0 [32]. 


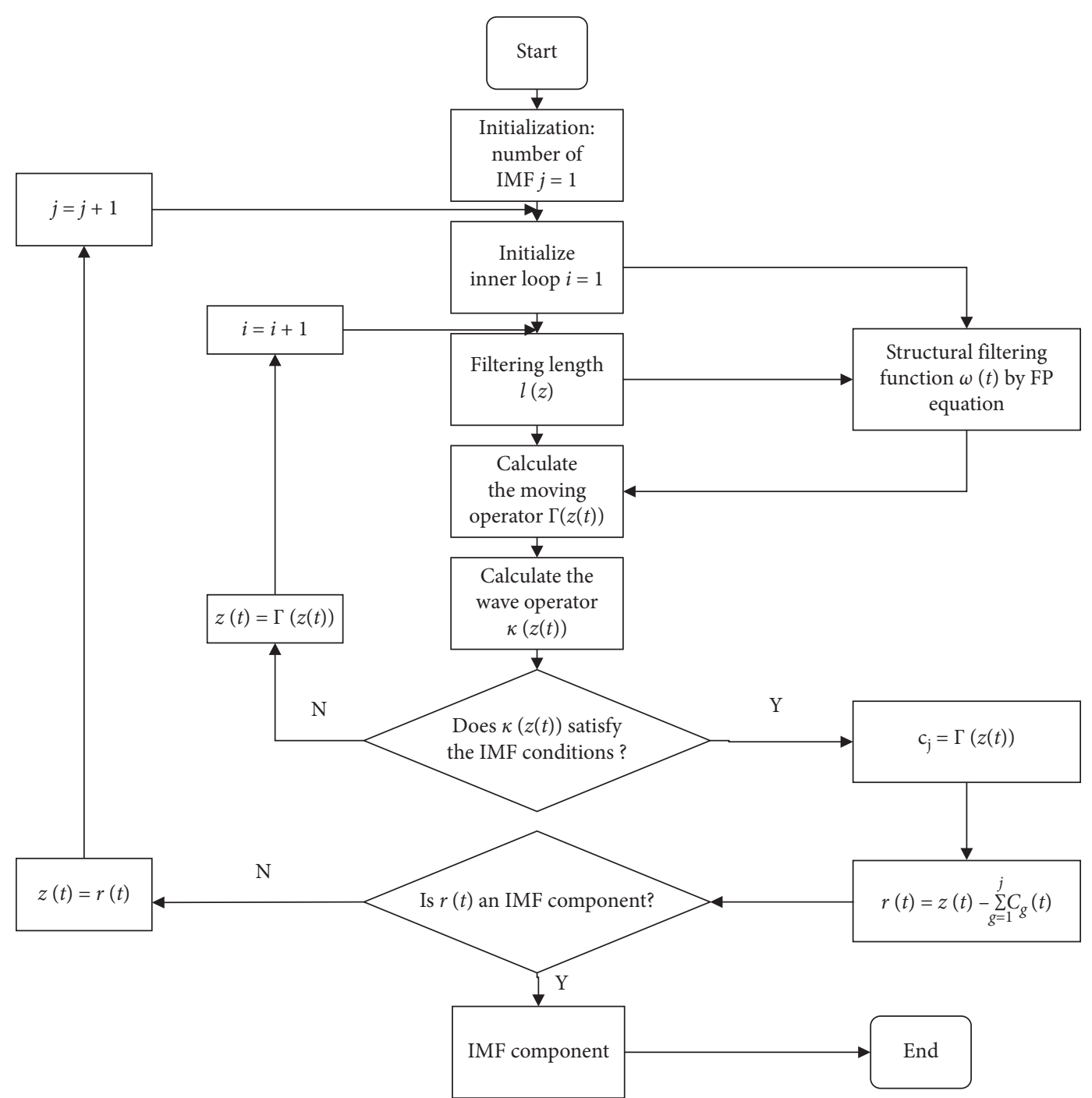

Figure 1: Process of outer loop.

\section{Proposed Methodology}

The hybrid method to diagnose 3D rotor eccentricity faults in synchronous generators based on ALIF PE and KFCM can be diagrammed in Figure 2. And the detailed process is introduced as follows.

Step 1. Decompose the signal data of training samples and testing samples using the ALIF method to obtain multiorder eigenmode components.

Step 2. Conduct correlation analysis between the original signal and the multiorder eigenmode components in order to get optimal mode components.

Step 3. Calculate the PE of the effective mode components as the feature vector so as to construct the training sample matrix and testing sample matrix.
Step 4. Input the training sample matrix to KFCM to obtain the center of clustering.

Step 5. Input the center of clustering and testing samples to KFCM together; then, we can get the result of clustering.

\section{Simulation and Experiment Study}

In this section, the simulating signal and the test data from the CS-5 prototype generator are employed to validate the proposed method. And, to get a comprehensive comparison, they are applied to some other popular methods including EEMD, CEEMDAN, and the clustering method based on FCM as well.

4.1. Application to Simulating Signal. The simulating signal is constructed as 


$$
x(t)=\sin (2 * 50 \pi t)+\sin \left(2 * 100 \pi t+\frac{\pi}{6}\right)+\sin \left(2 * 200 \pi t+\frac{\pi}{3}\right)+\eta(t),
$$

where $x(t)$ consists of three harmonic signals and the white Gaussian noise whose signal-to-noise ratio is $30 \mathrm{~dB}$. Figure 3 illustrates the time domain waveform of the simulating signal, with the number of the sampling points 1000 and the sampling frequency of $1 \mathrm{kHz}$.

The results after decomposing by EEMD and CEEMDAN are shown in Figures 4 and 5, respectively. It is suggested from Figure 4 that IMF3 indicates an obvious mode mixing due to the impact of the white Gaussian noise, while in Figure 5, it shows that IMF3 and IMF 4 both have the endpoint effect. Therefore, both EEMD and CEEMD have defects.

The signal is also decomposed by ALIF, as illustrated in Figure 6. It shows that there are three IMF components and a residual $r$. The components IMF1, IMF2, and IMF3 correspond to the three sinusoidal components of $200 \mathrm{~Hz}, 100 \mathrm{~Hz}$, and $50 \mathrm{~Hz}$, respectively. Obviously, the three IMF components have neither the mode mixing nor the endpoint effect, suggesting the superior effect of ALIF against the traditional CEEMDAN and EEMD methods.

4.2. Comparison of Entropy Algorithms. Herein, we calculated the PE, DE, AE, and SE of the eccentricity faults signal and normal signal, respectively. Based on the entropy of normal signal, we obtained the difference between axial eccentricity, radial eccentricity, mixed eccentricity signal, and normal signal under corresponding entropy, respectively. The result is shown in Figure 7.

The result shows that the difference of PE between the three fault conditions and normal signal is the most obvious and the values are $0.542,0.962$, and 1.369 , respectively. Therefore, it is easy to be distinguished based on the clear distribution. Finally, we chose PE as the indicator to detect the eccentricity faults.

\subsection{Application to Experimental Signal}

4.3.1. Method to Test the Vibration Signal. The experiment is carried out on the CS-5 prototype generator which has one pole-pair and a rated rotation of $3000 \mathrm{rpm}$, as shown in Figure $8(\mathrm{a})$. The rotor is kept stable with the foundation by the bearing blocks, while the stator can be moved along the radial direction and axial direction, respectively, to simulate the radial eccentricity, the axial eccentricity, and the mixed eccentricity, as illustrated in Figure 8(b). The movements are performed by 8 screws, with four for the radial and the other four for the axial shifts, and controlled by four dial indicators.

During the experiment, the excitation current was set to 2.5 A to output the phase voltage at $220 \mathrm{~V}$, with three sliding rheostats of $500 \Omega$ as the loads for the three phases. The vibration signals of the rotor and the stator are sampled through four accelerometers whose sensitivity is $10 \mathrm{mV} /$ $\mathrm{mm} / \mathrm{s}$, with two for the radial vibration and the other two for the axial vibration, as illustrated in Figure 8(c). The sampling frequency was set to $5000 \mathrm{~Hz}$.

Four groups of experiments were taken, namely, the normal condition, the radial eccentricity of $0.2 \mathrm{~mm}$ (the radial air-gap length is $1.2 \mathrm{~mm}$ ), the axial eccentricity of $2 \mathrm{~mm}$, and the mixed eccentricity composed of the former two.

4.3.2. Fault Classification. EEMD, CEEMDAN, and ALIF methods are employed to decompose the vibration data, respectively. Taking the radial rotor vibration in the axial eccentricity case as an example, the decomposed results are illustrated in Figure 9. It shows that the results of EEMD and CEEMDAN are somewhat similar. The components E4, E5, $\mathrm{C} 5$, and $\mathrm{C} 6$ all indicate obvious mode mixing. However, the frequency range occupied by the first six components decomposed by ALIF is significantly refined, without any frequency mixing problem (E1 E6, C1 C6, and A1 A6 indicate amplitudes of mode components).

Since the IMF component with large correlation coefficient is able to well retain the fault features of the signal [32], in this paper, the correlation coefficients between the IMF components and the original signal are calculated, as listed in Table 1.

As indicated in Table 1, the correlation coefficients between the first three IMF components and the original signal are far greater than those of the other components. Therefore, it can be considered that most of the fault information is included in the first three IMF components. Consequently, IMF1 to IMF3 are selected to represent the original signal. In this paper, $\mathrm{PE}$ is used to extract the fault features of the IMF components for further clustering. The ALIF PE of the four signal types is calculated, respectively, as shown in Table 2.

Besides, we need to set delay time $\tau$ and dimension $m$ to calculate PE. According to the change of PE at different delay times in Figure 10, it can be seen that the delay time $\tau$ has little influence on the calculation of time series. However, if the dimension $m$ is too small, it is difficult to detect the dynamic mutation of the time series because there are too few states in the reconstructed sequence; if the value of $m$ is too large, the calculation time is too long to reflect the subtle transformation of the time series. According to Bandt's suggestion [21], the value of $m$ is $3 \sim 7$. Combined with another research $[33,34]$, we set $m=6$, $\tau=1$, and a relatively good clustering effect is obtained.

As indicated in Table 2, the PE values of IMF1 to IMF3 are varied, suggesting that the complexities of them are different. Consequently, the eigenvectors composed of these permutation entropies should be also different, offering the possibility of hard classification.

\section{Case Verification and Result Analysis}

5.1. Case Setup. 20 groups of vibration data for each case (normal, radial eccentricity, axial eccentricity, and mixed 


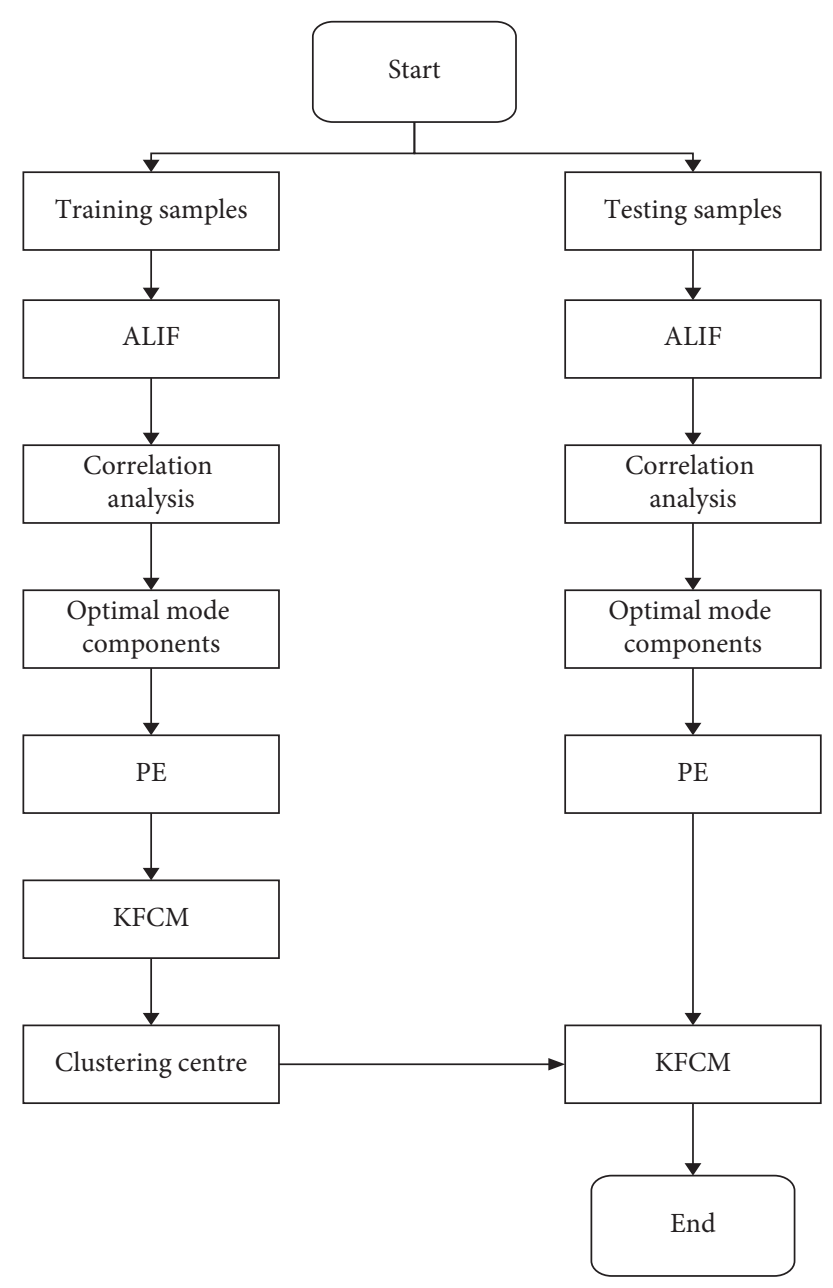

FIgure 2: Process of the proposed method.

eccentricity) are taken continuously (10 groups for training and 10 groups for testing), and the points of each set are 2048.

EEMD, CEEMDAN, and ALIF methods are used to adaptively decompose the 20 sets of data and obtain the multiorder IMF components. Then, the correlation coefficients between each IMF and the original signal are calculated.

By calculating the PE of the first three IMF components, the eigenvector matrixes are obtained, with the forms of $3 \times 40$.

Firstly, four clustering centers are obtained by clustering the eigenvector matrixes. Then the clustering centers as well as the eigenvector matrixes are applied with KFCM. Calculations are performed iteratively until the errors are less than the tolerance. The final results are illustrated in Figure 11.

5.2. Results Discussion. As illustrated in Figure 11(a), the data points are scattered around the cluster center. Moreover, the mixed eccentricity and the radial eccentricity faults cannot be distinguished. Therefore, EEMD does not offer a satisfactory effect.
Figure 11(b) shows the clustering effect of CEEMDAN. It is shown that the signal data points are clustered around the center, with the distribution compact. Compared with Figure 11(a), the clustering effect of this method is better than that of EEMD. It can basically realize the detection and classification of different eccentricity faults.

Figure 11(c) shows the effect of KFCM clustering based on ALIF. It is shown that the clustering centers of each case are far apart from each other and therefore can be distinguished clearly. What is more, the distances between two cluster centers are larger than those of the aforementioned two methods, showing an even better effect.

In order to compare the clustering effect of the above three algorithms more accurately, the classification coefficient $S$ and the average FE $E$ are employed, as shown in Table 3.

According to [32], the clustering effect of samples will be better if the classification coefficient $S$ is closer to 1 and the average FE $E$ is closer to 0 . Obviously, the proposed method has the best clustering effect, while EEMD ranks the last.

In order to verify the superiority of the proposed method, the aforementioned eccentricity fault data are further analyzed for comparison with that FCM. The clustering result by ALIF-FCM is illustrated in Figure 11(d). 


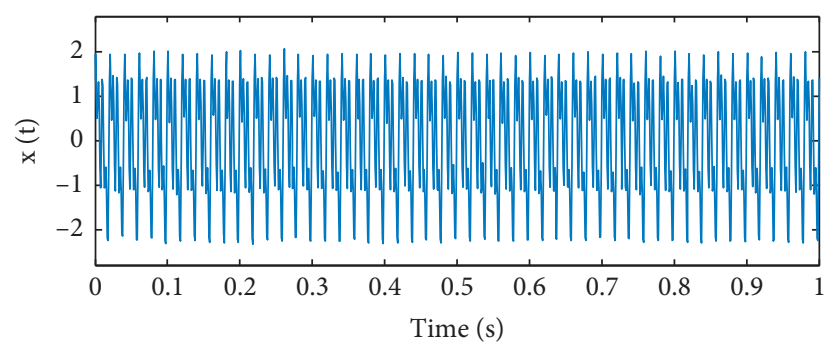

FIgURE 3: Simulating signal.
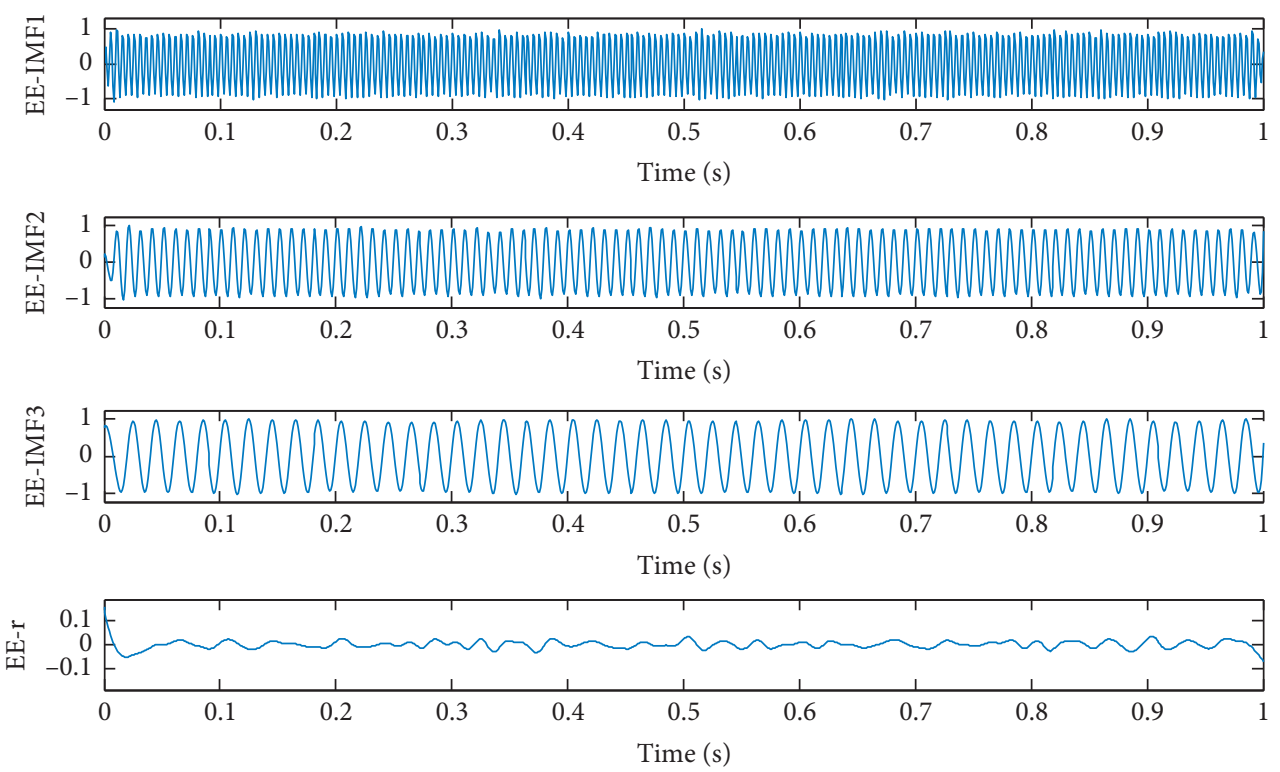

Figure 4: EEMD result of the simulating signal.
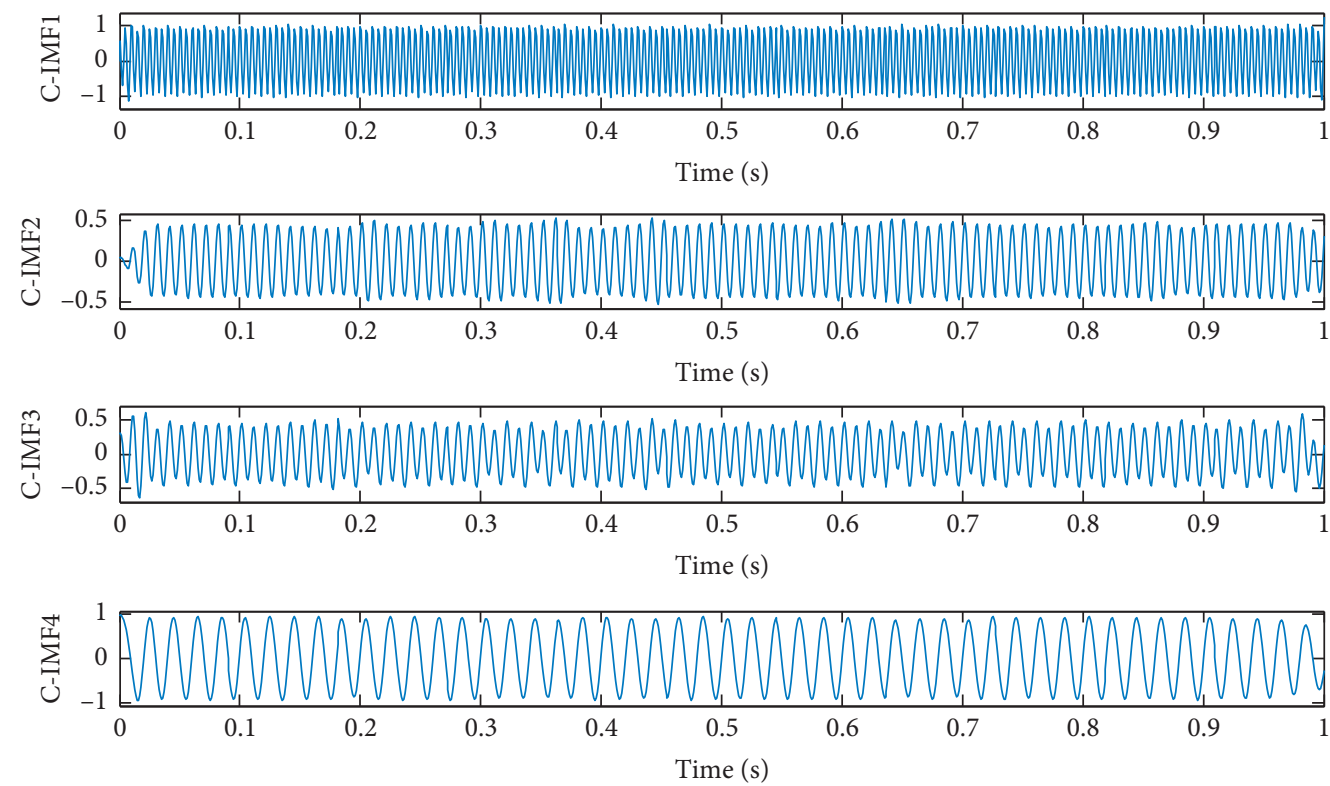

Figure 5: CEEMDAN result of the simulating signal.

As indicated in Figure 11(d), the mixed eccentricity and the normal operation signals are far away. The radial eccentricity and axial eccentricity fault signals are relatively close while the distinction is clear. That means this method can realize the identification and division of different kinds of eccentricity faults. However, comparing 

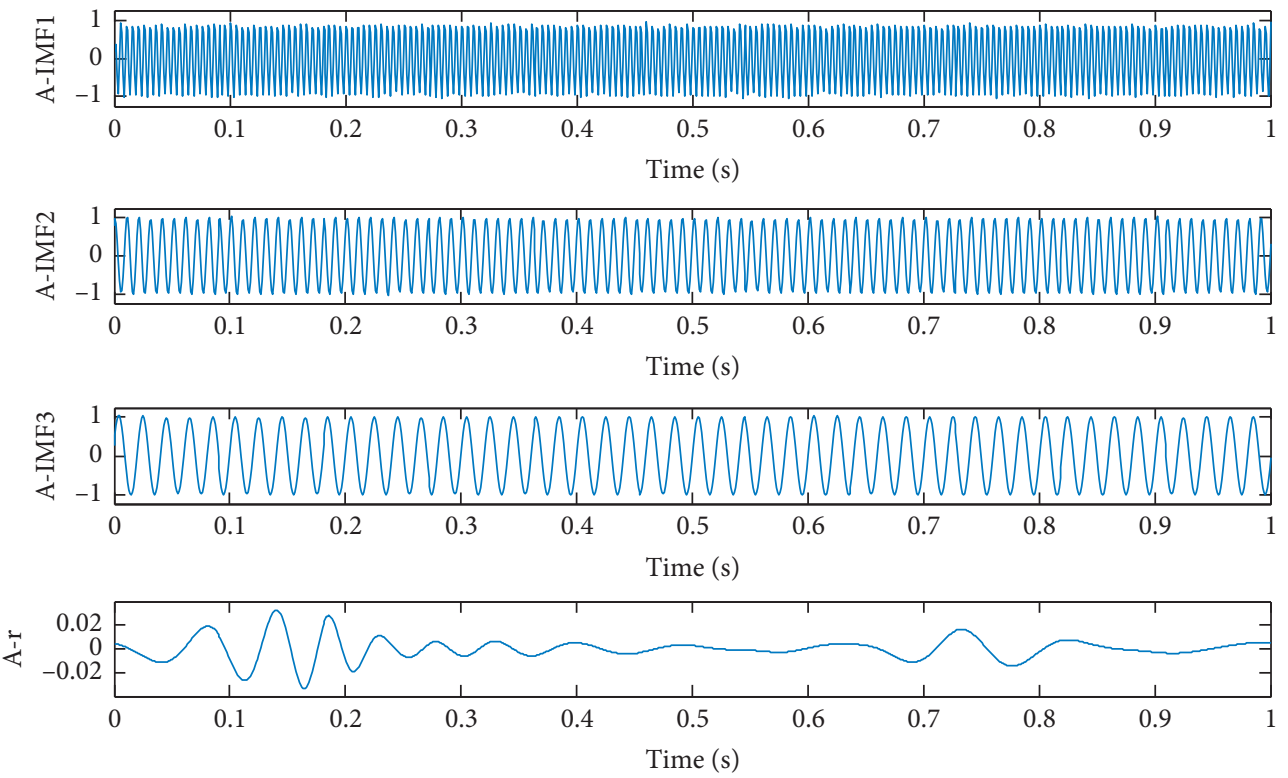

FIgURE 6: ALIF result of the simulating signal.

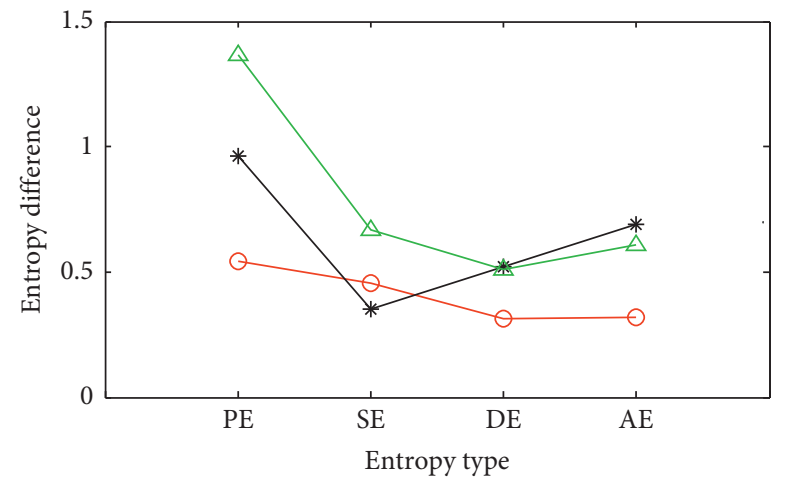

- Axial difference

* Radial difference

$\triangle$ Mixed difference

Figure 7: The differences of entropy.
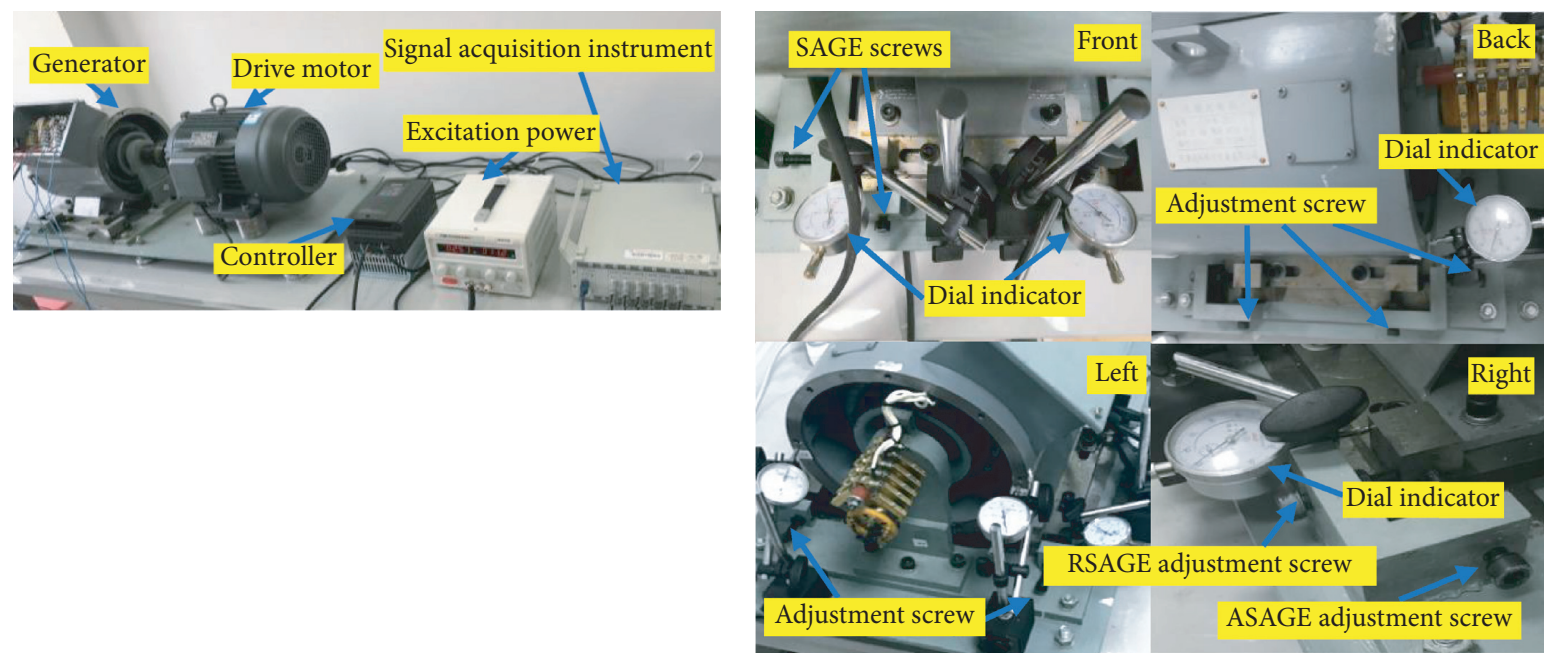

(a)

(b)

Figure 8: Continued. 


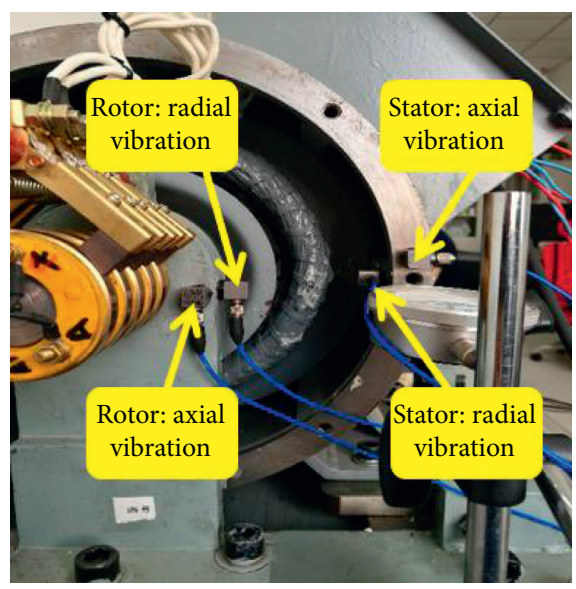

(c)

Figure 8: Method to test the vibration signal, (a) picture of experiment rig, (b) method to set radial and axial eccentricity, and (c) accelerometer setup.
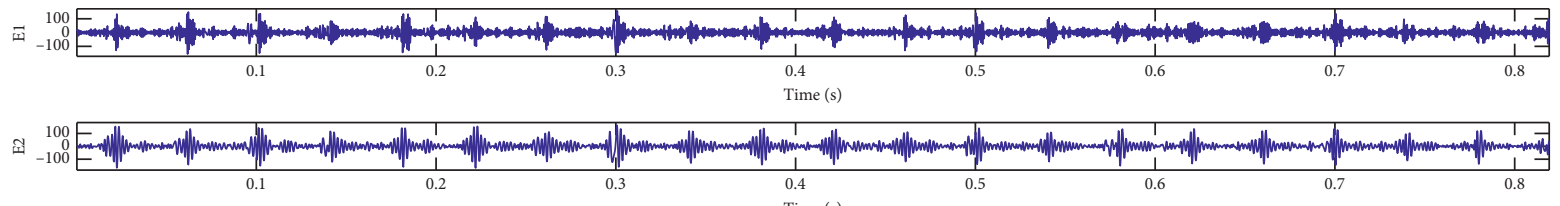

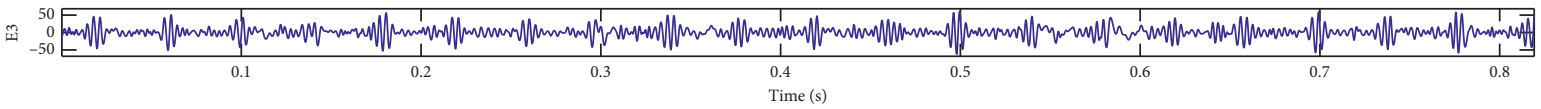

-100

(1)

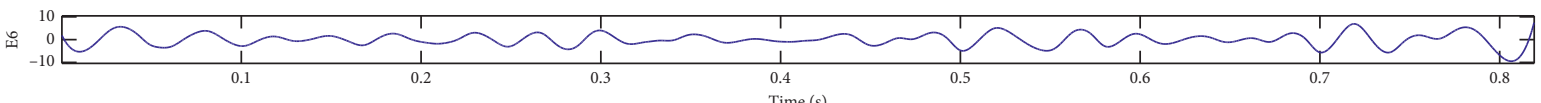

(a)
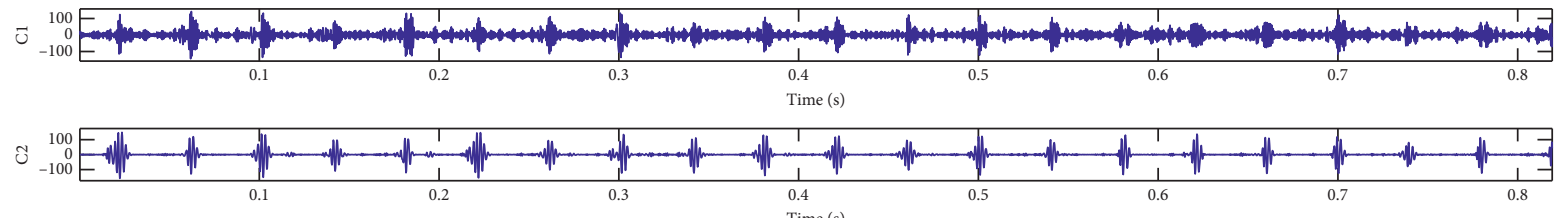

$8 \int_{-50}^{50} \underbrace{5}_{0.1}$

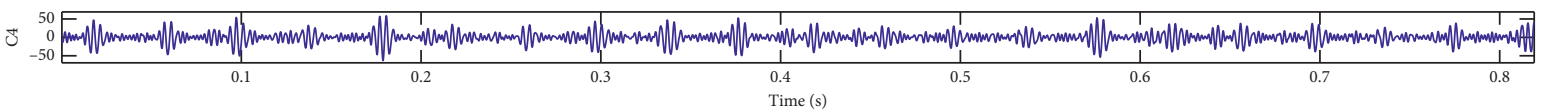

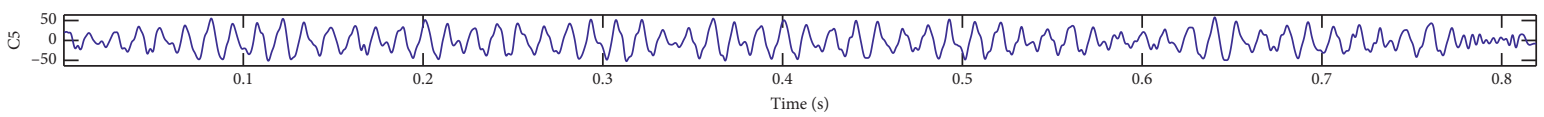

(50)

(b)

FIgURE 9: Continued. 

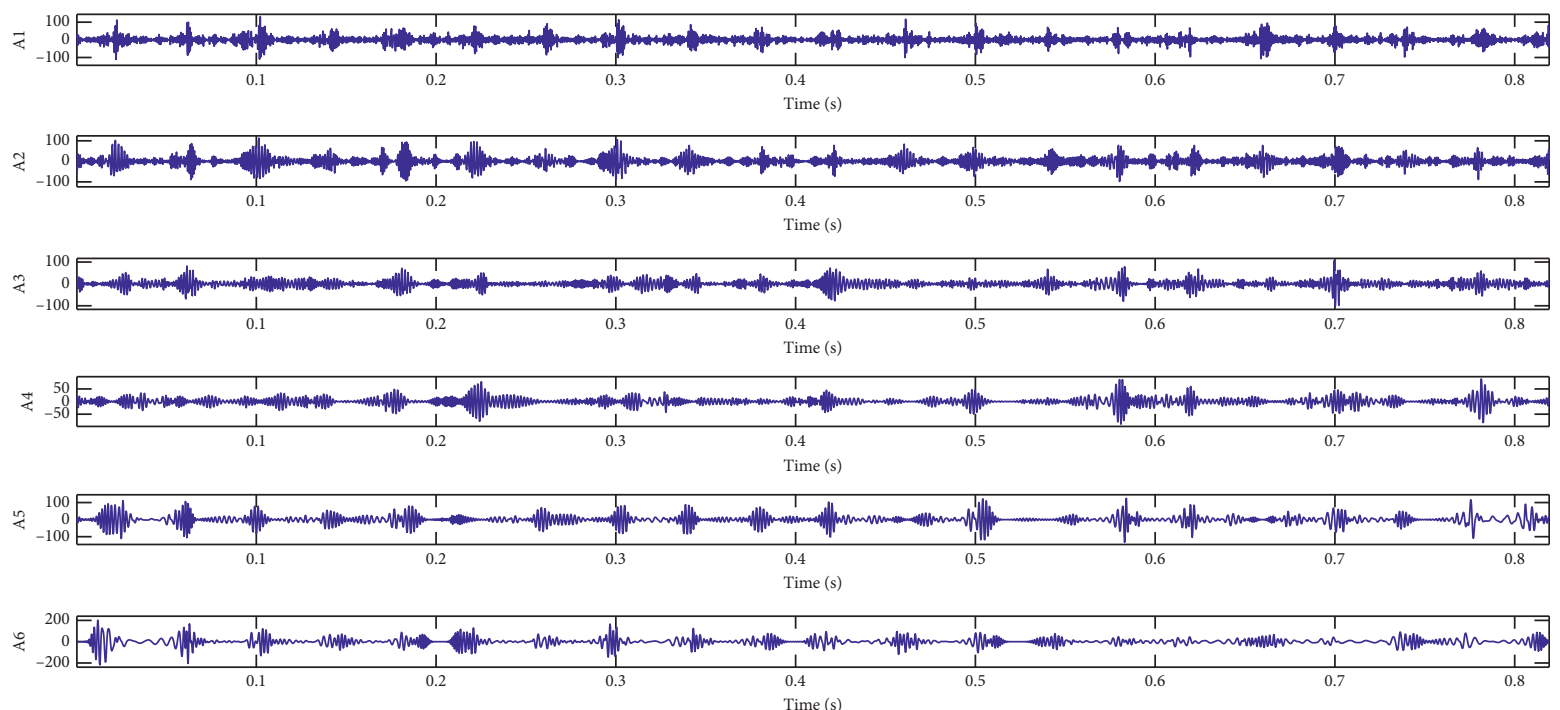

(c)

FIGURE 9: Decomposing results by three different methods, (a) signal decomposed by EEMD, (b) signal decomposed by CEEMDAN, and (c) signal decomposed by ALIF.

TABle 1: Correlation coefficients between IMF components and the original signal.

\begin{tabular}{lcccrr}
\hline \multirow{2}{*}{ Algorithm } & \multicolumn{3}{c}{ Correlation coefficient } & IMF4 & IMF5 \\
\hline EEMD & IMF1 & IMF2 & IMF3 & 0.2253 & 0.0328 \\
CEEMDAN & 0.7942 & 0.3723 & 0.2661 & 0.0623 & 0.0326 \\
ALIF & 0.7826 & 0.4265 & 0.2984 & 0.0927 & 0.0658 \\
\hline
\end{tabular}

TABLE 2: Permutation entropy of the first three IMF components.

\begin{tabular}{lccr}
\hline Signal type & & Permutation entropy & IMF2 \\
\hline Normal & IMF1 & 2.7183 & 2.6223 \\
Radial eccentricity & 4.1653 & 5.0729 & 4.9135 \\
Axial eccentricity & 5.7926 & 5.9557 & 5.4241 \\
Mixed eccentricity & 6.0456 & 5.1811 & 4.8916 \\
\hline
\end{tabular}

Figure 11(d) with 11(c), it is shown that the distribution of data points based on the KFCM clustering method is more compact, and the different signal intervals are more obvious.
In order to quantitatively compare the effects of FCM and KFCM, the classification coefficient $S$ and the average FE $E$ as well as the iteration times these two methods are calculated, as shown in Table 4. Obviously, although the KFCM 


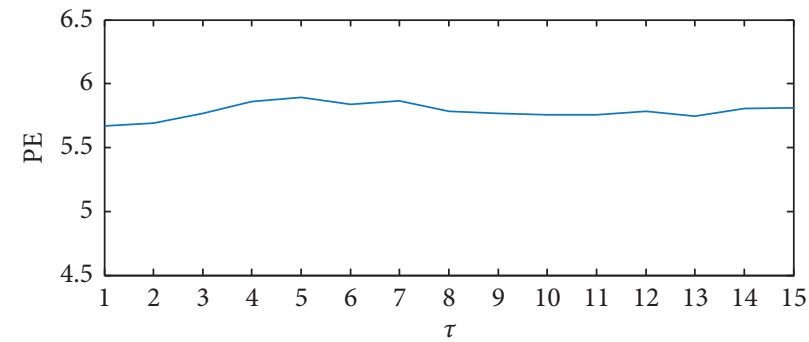

Figure 10: Permutation entropy (PE) at different delay times.
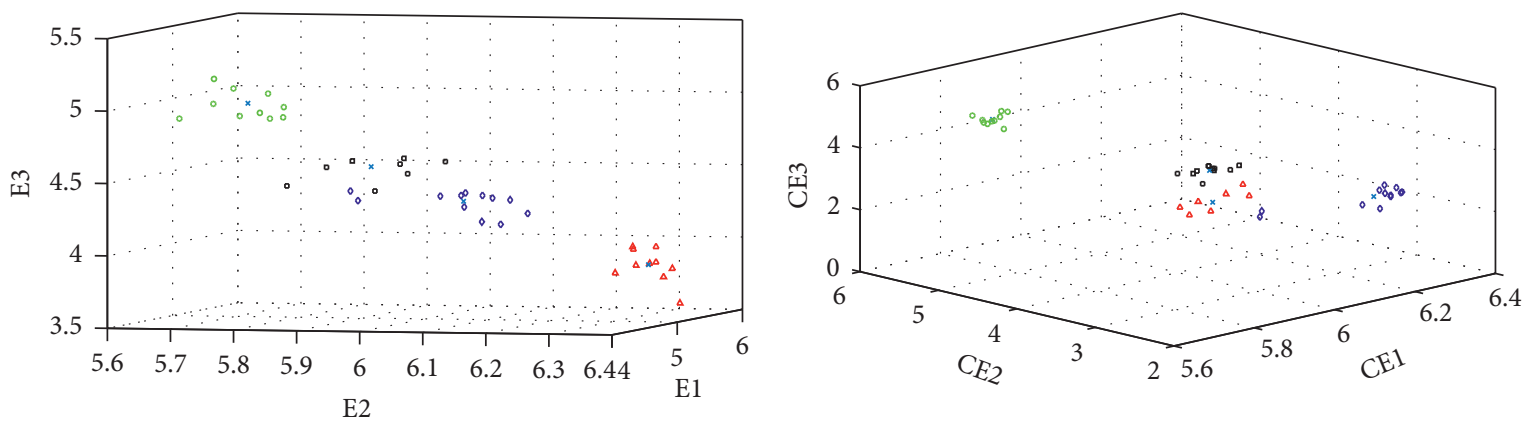

- Normal

- Mixed eccentricity

$\triangle$ Axial eccentricity * Center

- Radial eccentricity

(a)

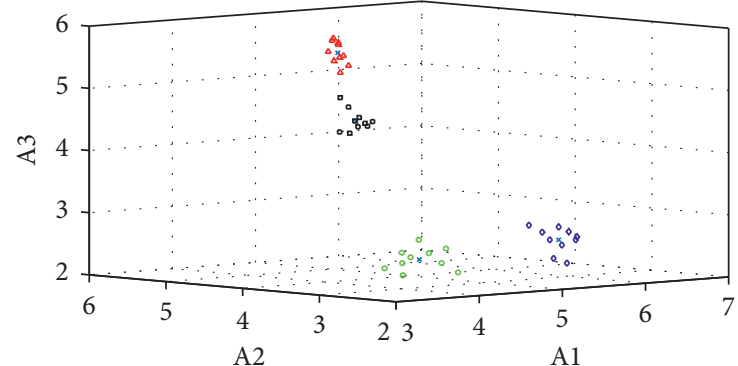

- Normal

$\triangle$ Axial eccentricity

- Radial eccentricity

- Mixed eccentricity

* Center

(c)
- Normal

$\triangle$ Axial eccentricity

- Radial eccentricity

- Mixed eccentricity

* Center

(b)

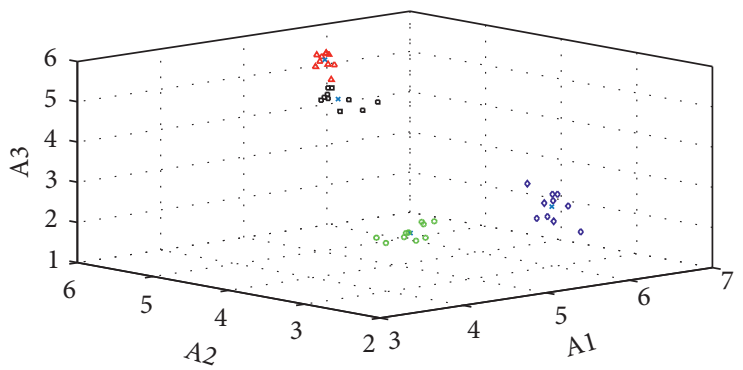

- Normal

- Mixed eccentricity

$\triangle$ Axial eccentricity

- Radial eccentricity
* Center

(d)

Figure 11: Cluster results by four different methods: (a) clustered by EEMD-KFCM, (b) clustered by CEEMDAN-KFCM, (c) clustered by ALIF-KFCM (this paper), and (d) clustered by ALIF-FCM.

TABle 3: Clustering index of different methods.

\begin{tabular}{lcc}
\hline Algorithm & Classification coefficient $S$ & Average fuzzy entropy $E$ \\
\hline EEMD & 0.7412 & 0.5186 \\
CEEMDAN & 0.8941 & 0.2188 \\
ALIF-KFCM & 0.9268 & 0.1758 \\
\hline
\end{tabular}

TABLE 4: Clustering index of KFCM and FCM.

\begin{tabular}{lccc}
\hline Algorithm & Classification coefficient $S$ & Average fuzzy entropy $E$ & Iteration times \\
\hline FCM & 0.9082 & 0.2067 & 12 \\
KFCM & 0.9268 & 0.1758 & 13 \\
\hline
\end{tabular}


based method has one more interaction, it has a superior clustering effect since its $S$ is closer to 1 while its $E$ is closer to 0 .

\section{Conclusion}

In this paper, we proposed a fault diagnosis method for the 3D eccentricity faults in synchronous generators based on ALIF PE and KFCM. This method employs ALIF, which has a better effect than EEMD and CEEMDAN in avoiding the influence of mode mixing and endpoint effect, to decompose the vibration signal. In the meantime, the proposed method employs the PE and KFCM, which are superior to EEMDKFCM, ALIF-KFCM, and CEEMDAN-KFCM in getting the classification coefficient $S$ and the average FE $E$, to classify the fault types.

The index factors of the clustering effect, namely, the classification coefficient $S$ and the average FE $E$ of ALIFKFCM, are 0.9268 and 0.1758 , respectively. Comparing with the results by EEMD-KFCM $(S=0.7412$ and $E=0.5186)$, the proposed ALIF-KFCM method improves $25.0 \%$ in $S$ (the closer to 1 , the better), while it improves $66.1 \%$ in $E$ (the closer to 0 , the better). Comparing with the results by CEEMDAN-KFCM $(S=0.8941$ and $E=0.2188)$, the proposed method improves $3.66 \%$ in $S$ and $19.7 \%$ in $E$. Comparing with the results by ALIF-FCM $(S=0.9082$ and $E=0.2067$ ), the proposed method improves $2.04 \%$ in $S$ and $14.9 \%$ in $E$. Obviously, the proposed method is superior to these aforementioned methods.

The proposed method presents a qualified detection and classification of the 3D eccentricity faults, which includes the radial eccentricity, the axial eccentricity, and the mixed eccentricity composed of the former two. We focused on the type of static eccentricity faults rather than the degree. Based on its clear figure and intelligent effect, this method is potential for the application in the eccentricity fault monitoring and diagnosis.

In our next step work, we will focus on how to decrease the iteration times so that the proposed method can be performed more efficiently. In addition, we will try to analyze the diagnosis method under dynamic eccentricity fault and even the complex fault conditions including both eccentricity and interturn short circuit.

\section{Data Availability}

The data used to support the findings of this study are available from the corresponding author upon request.

\section{Conflicts of Interest}

The authors declare that there are no conflicts of interest regarding the publication of this paper.

\section{Acknowledgments}

This work was supported by the National Natural Science Foundation of China (51777074), the Hebei Provincial Natural Science Foundation (E2020502032), the Fundamental Research Funds for the Central Universities
(2020MS114 and 2018YQ03), and the Top Youth Talent Support Program of Hebei Province ([2018]-27).

\section{References}

[1] Y.-L. He, W.-Q. Deng, G.-J. Tang, X.-L. Sheng, and S.-T. Wan, "Impact of different static air-gap eccentricity forms on rotor UMP of turbogenerator," Mathematical Problems in Engineering, vol. 2016, Article ID 5284815, 13 pages, 2016.

[2] Y. He, Y. Zhang, Y. Meng, G. Tang, W. Deng, and H. Zhong, "Enhanced detection of characteristic vibration signal of generator based on self-adapted multi-scale top-hat transformation," Recent Advances in Electrical \& Electronic Engineering, vol. 11, no. 1, pp. 418-424, 2018.

[3] Y.-L. He, W.-Q. Deng, B. Peng et al., "Stator vibration characteristic identification of turbogenerator among single and composite faults composed of static air-gap eccentricity and rotor interturn short circuit," Mathematical Problems in Engineering, vol. 2016, Article ID 5971081, 14 pages, 2016.

[4] X. Bao, "Review and prospect of air-gap eccentricity faults in induction machines," Proceedings of the CSEE, vol. 33, no. 6, pp. 93-100, 2013.

[5] T. Goktas, M. Zafarani, and B. Akin, "Discernment of broken magnet and static eccentricity faults in permanent magnet synchronous motors," IEEE Transactions on Energy Conversion, vol. 31, no. 2, pp. 578-587, 2016.

[6] I. Attoui and A. Omeiri, "Modeling, control and fault diagnosis of an isolated wind energy conversion system with a selfexcited induction generator subject to electrical faults," Energy Conversion and Management, vol. 82, pp. 11-26, 2014.

[7] N. E. Huang, Z. Shen, S. R. Long et al., "The empirical mode decomposition and the hilbert spectrum for nonlinear and non-stationary time series analysis," Proceedings of the Royal Society of London. Series A: Mathematical, Physical and Engineering Sciences, vol. 454, no. 1971, pp. 903-995, 1998.

[8] Z. Wu and N. E. Huang, "Ensemble empirical mode decomposition: a noise assisted data analysis method," Advances in Adaptive Data Analysis, vol. 1, no. 1, pp. 1-41, 2009.

[9] Z. Wu and N. E. Huang, "A study of the characteristics of white noise using the empirical mode decomposition method," Proceedings of the Royal Society A: Mathematical, Physical and Engineering Sciences, vol. 460, no. 2046, pp. 1597-1611, 2004.

[10] W. Xue, X. Dai, J. Zhu, Y. Luo, and Y. Yang, "A noise suppression method of ground penetrating radar based on EEMD and permutation entropy," IEEE Geoscience and Remote Sensing Letters, vol. 16, no. 10, pp. 1625-1629, 2019.

[11] M. E. Torres, M. A. Colominas, G. Schlott-Hauer et al., "A complete ensemble empirical mode decomposition with adaptive noise," in Proceedings of the IEEE International Conference on Acoustics Speech and Signal Processing (ICASSP 2011, pp. 4144-4147, Prague, Czech Republic, May 2011.

[12] N. Ji and L. Wang, "Bearing fault diagnosis based on fast spectral kurtosis graph algorithm aided by CEEMDAN," Mechanical Engineer, no. 1, pp. 76-79+82, 2020, in Chinese.

[13] A. Cicone, J. Liu, and H. Zhou, "Adaptive local iterative filtering for signal decomposition and instantaneous frequency analysis," Applied and Computational Harmonic Analysis, vol. 41, no. 2, pp. 384-411, 2016.

[14] G.-J. Tang and B. Pang, "Fault diagnosis of a turbo-generator rotor based on ALIF-HT," Journal of Chinese Society of Power Engineering, vol. 37, no. 11, pp. 883-889, 2017.

[15] J. S. Richman and J. Randall Moorman, "Physiological timeseries analysis using approximate entropy and sample 
entropy," American Journal of Physiology- Heart and Circulatory Physiology, vol. 278, pp. 2039-2049, 2000.

[16] L.W. Zhong, "Entropy theory of distributed energy for internet of things," Nano Energy, vol. 58, pp. 669-672, 2019.

[17] Z. Cao and C.-T. Lin, "Inherent fuzzy entropy for the improvement of EEG complexity evaluation," IEEE Transactions on Fuzzy Systems, vol. 26, no. 2, 2018.

[18] H. Zhao, M. Sun, and X. Yang, "A new feature extraction method based on EEMD and multi-scale fuzzy entropy for motor bearing," Entropy, vol. 19, no. 1, 2016.

[19] M. Rostaghi and H. Azami, "A measure for time-series analysis," IEEE Signal Processing Letters, vol. 23, no. 5, pp. 610-614, 2016.

[20] T. Wang, Research on Vibration Signal Feature Extraction Method of Generator under Interturn Short-Circuit Faults, Degree thesis of North China Electric Power University, Beijing, China, 2020.

[21] C. Bandt and B. Pompe, "Permutation entropy: a natural complexity measure for time series," Physical Review Letters, vol. 88, no. 17, Article ID 174102, 2002.

[22] W. Ding and Z. Zhang, "Fault identification method of transmission gear based on morphological wavelet and permutation entropy," Journal of Mechanical Transmission, vol. 43, no. 10, pp. 165-168, 2019.

[23] Y. Ren, S. Huangjin, L. Hu, X. Li, and H. Chen, "Research on denoising method of hydraulic turbine based on fourier decomposition and permutation entropy," Water Power, vol. 46, no. 10, pp. 96-99, 2020.

[24] J. Sun, J. Liu, and L. Zhao, "Clustering algorithms research," Journal of Software, vol. 19, no. 1, pp. 48-61, 2008.

[25] F. Wu and Y. Mao, "A novel fuzzy C-means algorithm based on k-d tree," Computer and Modernization, vol. 11, pp. 1-5, 2015.

[26] S. Sun, Q. Zhang, T. Du, J. Wang, and Y. Wang, "Study of evaluation method for low voltage conventional circuit breaker switching fault degree based on vibration signal," Proceedings of the CSEE, vol. 37, no. 18, pp. 5473-5482, 2017.

[27] F. Bi, D. Tang, L. Zhang, Li Xin, T. Ma, and Y. Xiao, "Diesel engine fault diagnosis algorithm based on VMD and KFCM," Journal of Vibration, Measurement \& Diagnosis, vol. 40, no. 5, pp. 853-858, 2020.

[28] X. Zhang, J. Zhou, J. Guo, Q. Zou, and Z. Huang, "Vibrant fault diagnosis for hydroelectric generator units with a new combination of rough sets and support vector machine," Expert Systems with Applications, vol. 39, no. 3, pp. 2621-2628, 2012.

[29] Y. Gao, X. Liu, J. Xiang, and H. Huang, "A hybrid of FEM simulations and generative adversarial networks to classify faults in rotor-bearing systems," ISA Transactions, vol. 108, pp. 356-366, 2021.

[30] S. Wang, J. Xiang, Y. Zhong, and H. Tang, "A data indicatorbased deep belief networks to detect multiple faults in axial piston pumps," Mechanical Systems and Signal Processing, vol. 112, pp. 154-170, 2018.

[31] Y. Chang, Air-Gap Eccentricity Diagnosis for Introduction Motors Based on Vibration Analysis, Degree thesis of Zhejiang University, Hangzhou, China, 2016.

[32] J. C. Bezdek, "Cluster validity with fuzzy sets," Cybernetics and Systems, vol. 3, no. 3, pp. 58-72, 1974.

[33] G. Rao, "Method for optimal determination of parameters in permutation entropy algorithm," Journal of Vibration and Shock, vol. 33, no. 1, pp. 188-193, 2014.
[34] D. Q. Zhang and S. C. Chen, "Clustering incomplete data using kernel based fuzzy C means algorithm," Neural Processing Letters, vol. 18, no. 3, pp. 155-162, 2003. 